\title{
Measurement of the x-ray dose-dependent glass transition temperature of structured polymer films by $x$-ray diffraction
}

\author{
Heilke R. Keymeulen, Ana Diaz, Harun H. Solak, ${ }^{\text {a) }}$ Christian David, Franz Pfeiffer, \\ Bruce D. Patterson, and J. Friso van der Veen \\ Paul Scherrer Institut, CH-5232 Villigen PSI, Switzerland \\ Mark P. Stoykovich and Paul F. Nealey \\ Department of Chemical and Biological Engineering, University of Wisconsin-Madison, \\ Madison, Wisconsin 53706
}

(Received 14 January 2007; accepted 29 May 2007; published online 12 July 2007)

The glass transition temperature $\left(T_{g}\right)$ of polymer nanostructures was measured using a technique based on synchrotron x-ray diffraction from periodic grating structures. Poly(methyl methacrylate) (PMMA) nanostructures consisting of 1:1 lines:spaces with a $100 \mathrm{~nm}$ period and $100 \mathrm{~nm}$ height were characterized to have a $T_{g}$ of $118{ }^{\circ} \mathrm{C}$, which is comparable to the $T_{g}$ of PMMA in bulk systems. The $T_{g}$ of the PMMA structures also was measured as a function of absorbed x-ray dose. Doses ranging from 0 to $2400 \mathrm{~mJ} / \mathrm{mm}^{3}$ were delivered to the PMMA structures prior to the $T_{g}$ measurements; the $T_{g}$ of the structures was found to decrease from $118{ }^{\circ} \mathrm{C}$ to $95{ }^{\circ} \mathrm{C}$, respectively. The dose dependence of the PMMA glass transition temperature can be attributed to changes in the polymer molecular weight under exposure to x rays. (C) 2007 American Institute of Physics.

[DOI: $10.1063 / 1.2752548]$

\section{INTRODUCTION}

The glass transition temperature $\left(T_{g}\right)$ is a key parameter for determining the suitability of thermoplastic polymers for applications at both the macro- and nanoscale. ${ }^{1}$ Polymers are glasslike at temperatures below the $T_{g}$ but become rubbery with drastically different rheological and mechanical properties at temperatures above the $T_{g}$. The widespread application of polymers in coatings, membranes, and nanocomposites has necessitated an improved understanding of the thermophysical properties of polymers in confined geometries and in close proximity to surfaces and interfaces. Consequently, the glass transition temperature of thin polymer films has been measured using techniques such as ellipsometry, ${ }^{2-6}$ Brillouin light scattering, ${ }^{3,7}$ x-ray reflectivity, ${ }^{8}$ local thermal analysis, ${ }^{5,6,9}$ ensemble fluorescence, ${ }^{10,11}$ and quartz crystal microbalance measurements. ${ }^{12}$ Ultrathin polymer films with thicknesses less than $\sim 50 \mathrm{~nm}$ have been observed to have glass transition temperatures that deviate from the $T_{g}$ measured for the bulk polymer. ${ }^{2-12}$ These deviations often depend upon the film thickness, ${ }^{2-12}$ polymer molecular weight, ${ }^{4}$ the polymersubstrate and polymer-free surface interactions, ${ }^{3,5,13}$ and whether the film is supported or unsupported. ${ }^{3}$ Mechanical properties ${ }^{14-17}$ diffusion coefficients, ${ }^{10,18,19}$ and the physical aging behavior ${ }^{20-22}$ of polymers have shown similar dimension-dependent behavior in thin films.

Characterization of the glass transition of polymers in three-dimensional nanoscale structures is of critical importance to many nanofabricated systems. For example, polymeric photoresists are used in advanced lithography to pattern semiconductor devices at dimensions of $65 \mathrm{~nm}$ and

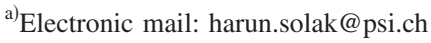

smaller, and the fabricated photoresist structures must be stable, both thermally and mechanically, when subjected to a wide range of processing temperatures and conditions. ${ }^{23-25}$ Three-dimensional nanostructures have substantially larger ratios of polymer-free surface to polymer-substrate interfacial areas than thin films and as a consequence have been measured to have $T_{g}$ 's that differ by as much as $15{ }^{\circ} \mathrm{C}$ from thin films of the same thickness. ${ }^{26}$ Furthermore, polymer nanostructures such as those lithographically patterned in complex layouts for integrated circuits may exhibit spatially varied glass transition temperatures as a function of the geometry, dimension, and local environment of the structures. The thermophysical behavior of polymers structured in three dimensions at the nanoscale must therefore be directly measured for the dimensions and geometries of interest. Many techniques used to determine the $T_{g}$ of polymers in thin films, however, are difficult or impossible to employ on structured systems. Brillouin light scattering is an exception that has been used to measure the elastic constants of periodic grating structures in polymer thin films. ${ }^{27-29} \mathrm{X}$-raybased methods for characterizing nanostructured polymers also are being pursued because of their high spatial resolution, the ability to operate in ambient conditions (i.e., vacuum conditions are not required, unlike electron microscopy), and the potential of probing buried structures. Smallangle $\mathrm{x}$-ray scattering (SAXS) and x-ray crystallography methods for nanoscale structural analysis must be adapted for the requirements of polymer materials and threedimensional structures. Recently, Jones et al. ${ }^{30}$ have introduced a transmission SAXS approach and characterized the periodicity, linewidth, and sidewall angle geometry of polymer gratings with nanometer precision.

Here, we report the development of a grazing incidence $\mathrm{x}$-ray diffraction technique for characterizing the glass tran- 


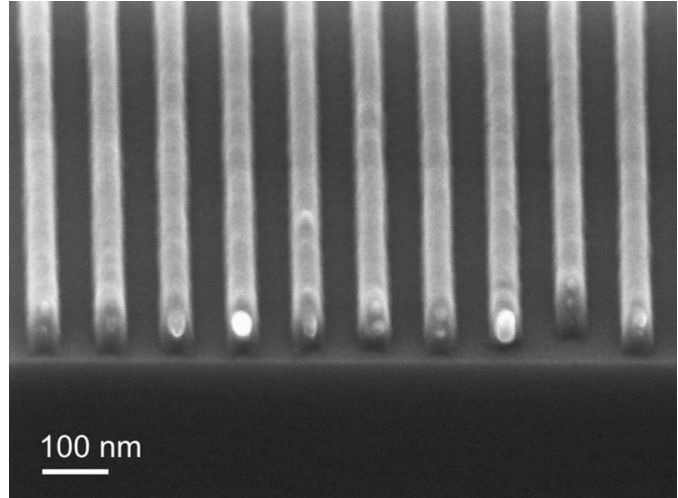

FIG. 1. Cross-sectional SEM image of a PMMA grating. The height of the grating lines is $100 \mathrm{~nm}$ and the gap between the grating lines is $50 \mathrm{~nm}$. The period is $100 \mathrm{~nm}$.

sition temperature of three-dimensional polymer nanostructures. A poly(methyl methacrylate) (PMMA) film structured with $100 \mathrm{~nm}$ period linear gratings was illuminated with a collimated x-ray beam and the diffracted intensity was measured as the temperature of the film was increased at a constant rate. The total diffracted intensity was found to be a useful parameter to determine the $T_{g}$ of the polymer nanostructures. The $T_{g}$ of the PMMA structures was measured to be $118{ }^{\circ} \mathrm{C}$ and corresponded well with values for PMMA measured in comparable bulk and thin-film systems. A linear decrease in the $T_{g}$ of the nanostructures as a function of the absorbed x-ray dose also was observed and quantitatively explained in terms of a decrease in the molecular weight of the PMMA polymer.

\section{EXPERIMENT}

The PMMA nanostructures were fabricated by photolithography in $100 \mathrm{~nm}$ thick PMMA films with extremeultraviolet interference lithography (EUV-IL) exposures at the XIL beamline of the Swiss Light Source (SLS) at the Paul Scherrer Institut (PSI), Switzerland. ${ }^{31,32}$ Figure 1 shows a cross-sectional scanning electron microscope (SEM) image of such a PMMA grating. The grating nanostructures had a height $H$ of $100 \mathrm{~nm}$, a period $T$ of $100 \mathrm{~nm}$, and a channel width $D$ of $50 \mathrm{~nm}$ and were fabricated over an area of 0.5 $\times 1.0 \mathrm{~mm}^{2}$. Poly(methyl methacrylate) with a molecular weight of $M_{w}=600 \mathrm{~kg} / \mathrm{mol}$ dissolved in chlorobenzene was purchased from Allresist GmbH, Germany. PMMA films with a thickness of $100 \mathrm{~nm}$ were prepared by spin-coating from solution on bare Si (100) wafers. No special cleaning or preparation steps were performed on the $\mathrm{Si}$ wafers prior to deposition. The films were annealed at $170{ }^{\circ} \mathrm{C}$ for $3 \mathrm{~min}$ on a hot plate after spin-coating. A linear grating structure was produced by coherently interfering two beams of $92 \mathrm{eV}$ energy and exposing the PMMA photoresist on the wafer to the interference pattern. $^{31,32}$ The final PMMA structures were formed after development for $30 \mathrm{~s}$ in a 3:7 solution of $\mathrm{H}_{2} \mathrm{O}$ :IPA (isopropyl alcohol).

$\mathrm{X}$-ray diffraction experiments on the gratings were performed at the materials science wiggler beamline X04SA of the SLS, using the reflection geometry shown in Fig. $2 .^{33}$ The photon energy of the collimated beam was $8.5 \mathrm{keV}$. The

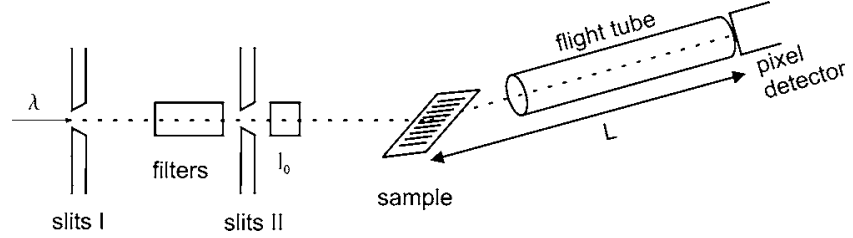

FIG. 2. Experimental setup for x-ray diffraction from a grating made by EUV-IL. The distance $L$ is $1.5 \mathrm{~m}$. The wavelength $\lambda$ of the incoming $\mathrm{x}$-ray beam is $0.146 \mathrm{~nm}$. Two pairs of slits define the beam. An ionization chamber counts the number of photons per second, $I_{\text {inc }}$, on the sample.

PMMA grating was oriented such that the grating lines were in the plane of incidence as shown in the figure. The $1.0 \mathrm{~m}$ long flight tube, filled with $\mathrm{He}$, was needed to reduce scattering from the air (the 1/ $e$ attenuation length of $\mathrm{x}$ rays in air at this energy is $1.4 \mathrm{~m}$ ). The $2 \mathrm{D}$ pixel detector consisted of $157 \times 366$ pixels, each $217 \times 217 \mu \mathrm{m}$ in size. The pixel detector used single-photon counting technology and provided unsurpassed signal-to-noise ratios. ${ }^{34}$ The distance $L$ from the sample to the detector was $1.5 \mathrm{~m}$, implying a subtended resolution angle of $1.45 \times 10^{-4}$ by $1.45 \times 10^{-4}$ rad per pixel. A grazing incidence angle $\theta_{i}$ of $1.0^{\circ}$, significantly higher than the critical angles for PMMA and $\mathrm{Si}$ at this energy $\left(0.156^{\circ}\right.$ and $0.211^{\circ}$, respectively) for total reflection, was used. The height $H$ of the grating lines $(100 \mathrm{~nm})$ corresponds to an effective height $H_{\text {eff }}$ of $2 H / \theta_{i}=11.4 \mu \mathrm{m}$ for the total path length traveled by the x-ray beam within the PMMA layer. The phase shift through the grating, $\phi$, is equal to $2 \pi \delta_{\text {PMMA }} H_{\text {eff }} / \lambda=1.82 \mathrm{rad}$. The fact that the phase shift is smaller than $\pi$ is important in the interpretation of the data, as we will explain below.

\section{RESULTS}

Diffraction patterns were monitored as a function of temperature. Figure 3 plots several diffraction measurements at different temperatures from $65^{\circ} \mathrm{C}$ to $117.6^{\circ} \mathrm{C}$. Two effects can be clearly seen: (i) from $65^{\circ} \mathrm{C}$ to $116^{\circ} \mathrm{C}$ the in-



FIG. 3. (Color online) Diffraction patterns of a PMMA grating with a period $T=100 \mathrm{~nm}$. The temperature was ramped from $65^{\circ} \mathrm{C}$ to $117.6^{\circ} \mathrm{C}$. A clear enhancement of the intensities in the diffraction peaks is seen up to the temperature of about $115^{\circ} \mathrm{C}$. Above $116^{\circ} \mathrm{C}$ the peaks disappear due to a glass transition. Note that the diffraction intensities have been offset for clarity. 
tensity in the diffraction peaks shows small variations with some enhancement observed especially in the higher order peaks; (ii) above $116^{\circ} \mathrm{C}$ the diffraction orders decrease quickly and disappear. The first effect can be qualitatively explained as being due to the annealing of the polymer gratings, i.e., the grating lines become smoother by the removal of defects such as surface roughness. The rapid disappearance of the diffraction pattern, however, was due to the glass transition, which causes the nanometer-scale PMMA grating to lose its structure and turn into a uniform film. Although the distribution of diffracted $\mathrm{x}$ rays into the different diffraction orders contains detailed information about the profile of the grating lines, the total diffracted intensity appears to be a useful measure that is directly related to a basic structural parameter, namely the standard deviation of the height of the grating pattern. The total diffracted intensity $\gamma$ can be found from the experimental data by adding the intensity in all the peaks,

$$
\gamma=\frac{1}{I_{\text {inc }}} \sum_{m=1}^{N} I_{m},
$$

where $I_{\text {inc }}$ is the incident X-ray intensity and $N$ is the number of the maximum order measured. The efficiency of diffraction into various orders by a grating can be obtained by expanding the field at the exit plane of the grating into a Fourier series. Using kinematical theory of diffraction for calculating the field distribution at the exit of a grating, we can calculate the quantity $\gamma$ for a square grating with period $T$, gap size $D$, and height $H$ by subtracting the zeroth-order efficiency from the total power, giving ${ }^{35}$

$$
\gamma=1-\frac{1}{T^{2}}\left\{D^{2}+(T-D)^{2}+2 D(T-D) \cos \phi\right\} .
$$

Equation (2) is valid for a pure phase grating, which is a reasonable approximation when hard $\mathrm{x}$ rays are used to illuminate thin polymer films. For example, the total absorption in the PMMA film in our case is less than $1 \%$. With straightforward manipulation one can show that, if the phase shift $\phi$ is much smaller than $\pi$, the total diffracted intensity is approximately equal to the variance of the phase shift imparted by the grating lines to the beam, i.e.,

$$
\gamma=\sigma_{\phi}^{2}
$$

where

$$
\sigma_{\phi}^{2}=\frac{(T-D) D}{T^{2}} \phi^{2} .
$$

The relation between the diffracted power and the variance of the grating structure is akin to the well-known dependence of scattering on the root-mean-squared roughness of a surface.

We performed diffraction experiments on a number of identical gratings that were pre-exposed to $\mathrm{x}$ rays for certain durations of time. The pre-exposures were performed at the same $\mathrm{x}$-ray diffraction setup just prior to the $\mathrm{x}$-ray diffraction measurements. The total $x$-ray dose delivered to the sample in the pre-exposure step was always significantly larger than the dose delivered during the diffraction measurement so that

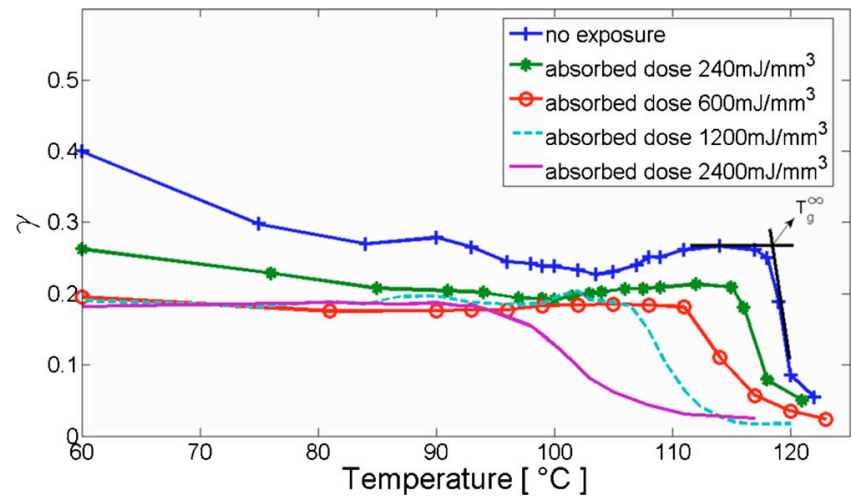

FIG. 4. (Color online) The total scattering power $\gamma$ of the grating as a function of temperature. Curves are shown for gratings which have been pre-exposed to $\mathrm{x}$ rays so as to study the influence of $\mathrm{x}$-ray radiation on the glass transition temperature $T_{g}$ of PMMA.

modification of the polymer during the measurement itself is negligible. This was accomplished by the introduction of strong attenuators into the beam during the measurements. The irradiation of PMMA is known to cause chain-scission and cross-linking reactions in the polymer which should significantly modify the glass transition temperature. Figure 4 plots $\gamma$ versus the temperature for several gratings preexposed with different $\mathrm{x}$-ray doses. The doses were calculated from the measured flux of the x-ray beam. The number of photons hitting the sample was measured with an ionization chamber to be $2.3 \times 10^{12}$ photons $/ \mathrm{s} / \mathrm{mm}^{2}$. Considering the angle of incidence ( $5^{\circ}$ during pre-exposures) and the absorption length of PMMA at this energy, we find absorbed doses, $\varepsilon$, of $240,600,1200$, and $2400 \mathrm{~mJ} / \mathrm{mm}^{3}$, for exposure times of $120,300,600$, and $1200 \mathrm{~s}$, respectively. The pre-exposures were performed at a constant temperature of $60{ }^{\circ} \mathrm{C}$. The temperature ramp rate during $\mathrm{x}$-ray diffraction measurements was $0.3^{\circ} \mathrm{C} / \mathrm{s}$. The data acquisition time for a single diffraction spectra like the ones shown in Fig. 3 was $1 \mathrm{~s}$.

In Fig. 4 we notice that at temperatures below $100{ }^{\circ} \mathrm{C}, \gamma$ decreases slightly for the sample that was not pre-exposed and the one that received only a slight dose $\left(240 \mathrm{~mJ} / \mathrm{mm}^{3}\right)$ as the temperature was ramped. In addition, the initial value of $\gamma$ exhibits a significant dependence on the exposure dose, i.e., this value goes down from about 0.4 to 0.2 between the unexposed sample and the sample that received the highest dose. Both observations can be interpreted to be due to loss of mass by creation of volatile components during x-ray exposure. The mass loss is likely to be accelerated by the elevated temperature during the x-ray exposure. As the temperature is further increased, a sudden drop of the diffracted intensity is observed. The point at which this sudden transition starts can be estimated by the intersection points of lines that approximate the constant intensity and the falling edge as seen in Fig. 4. We interpret the onset of this transition as the glass transition temperature $T_{g}$, which is plotted as a function of the absorbed dose $\varepsilon$ in Fig. 5 .

\section{DISCUSSION}

The experimental value of $\gamma$ at a temperature well below $T_{g}$ can be compared with the expected theoretical value 


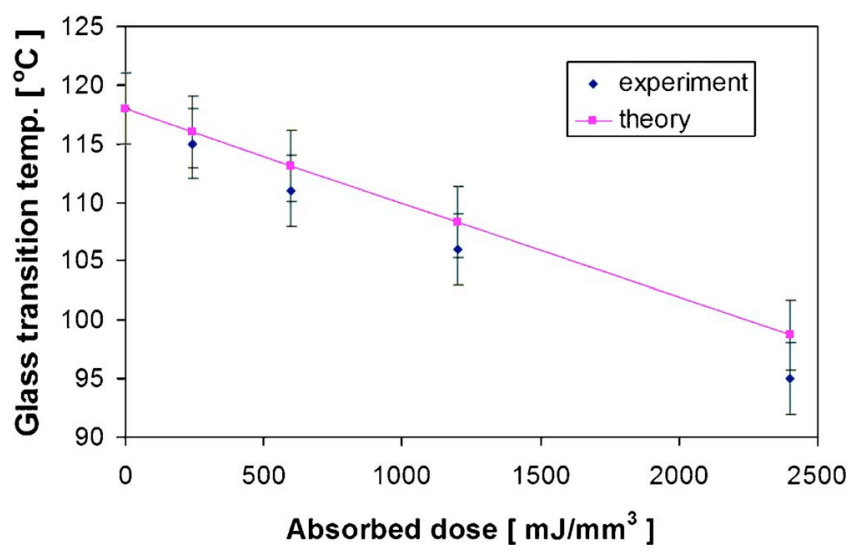

FIG. 5. (Color online) The glass temperature $T_{g}$ as a function of the absorbed $\mathrm{x}$-ray dose. The linear behavior can be explained with a theoretical model for the decrease in PMMA molecular weight as a function of $\mathrm{x}$-ray dose.

given by Eq. (2). For the nonexposed grating $\gamma$ is 0.4 at $65{ }^{\circ} \mathrm{C}$, whereas the theoretical value is 0.62 . In Fig. 4 we notice the large initial rate of decay of $\gamma$ for the nonexposed sample, which we have already attributed to x-ray induced mass loss. Before the first measurement, the sample was exposed to $\mathrm{x}$ rays for a short (undetermined) time for alignment purposes. Therefore, it is likely that the grating had already degraded to some extent before the first measurement. This is a very likely reason for the small difference between the theoretical and experimentally measured values of $\gamma$. In addition, the difference may be due to inaccuracies in the measurement of structural parameters (i.e., height and width of PMMA lines) by SEM or loss of diffracted intensity due to roughness in the gratings.

The effect of heat treatment on the shape of polymer micro- and nanostructures is well appreciated in the lithography community and is sometimes used to anneal out surface roughness. ${ }^{36}$ At temperatures just below the $T_{g}$ the shape change can show considerable variation depending on the properties of the polymer and the surface energy of the substrate. As the glass transition is reached, however, it is expected that the structured polymer will lose its shape and eventually will spread over the substrate. This dramatic transformation is accompanied by a corresponding decrease and eventual disappearance of the variance of the grating height, which is directly related to $\sigma_{\phi}$ and thus the diffracted power $\gamma$. Therefore, the prominent decrease in $\gamma$ observed in Fig. 4 at high temperatures can be attributed to the glass transition of the polymer.

The glass transition temperature $T_{g}$ of a polymer as a function of its molecular weight can be expressed by the Fox-Flory form,

$$
T_{g}=T_{g}^{\infty}-\frac{K}{M_{n}},
$$

where $T_{g}^{\infty}$ is the glass transition temperature for a polymer of infinite molecular weight and $K$ is the Fox-Flory constant. The latter is known from the literature to be $K=4.9$ $\times 10^{4} \mathrm{~g} \mathrm{~mol}^{-1}{ }^{\circ} \mathrm{C}$ for PMMA. ${ }^{37} M_{n}$ is the number averaged molecular weight, i.e., the weight of all the polymer molecules divided by the total number of polymer molecules.
TABLE I. Properties of PMMA (Refs. 37 and 39).

\begin{tabular}{ll}
\hline \hline$T_{g}^{\infty}$ & $118.0 \pm 3.0{ }^{\circ} \mathrm{C}$ \\
$K$ & $4.9 \times 10^{4} \mathrm{~g} \mathrm{~mol}^{-1}{ }^{\circ} \mathrm{C}$ \\
$M_{0}$ & $6.0 \times 10^{5} \mathrm{~g} \mathrm{~mol}^{-1}$ \\
$G$ & $1.9 \times 10^{-2} \mathrm{eV}^{-1}$ \\
$\rho$ & $1.19 \times 10^{6} \mathrm{~g} \mathrm{~m}^{-3}$ \\
\hline
\end{tabular}

For the PMMA gratings the pre-exposure to the $\mathrm{x}$-ray radiation alters the molecular weight of the polymer through the equation $^{38-40}$

$$
M_{n}=\frac{M_{0}}{1+g \varepsilon M_{0} /\left(\rho A_{0}\right)},
$$

where $M_{0}$ is the molecular weight of the unexposed PMMA, $g$ is the scission efficiency factor which is proportional to the radiation chemical yield, $\varepsilon$ is the absorbed energy density, $\rho$ is the density of PMMA, and $A_{0}$ is Avogadro's number. The radiation chemical yield of a polymer is a measure of the chemical action produced by radiation energy (expressed in number of molecules reacted per $100 \mathrm{eV}$ of absorbed energy). Combining Eqs. (5) and (6) yields

$$
T_{g}=K_{1}-K_{2} \varepsilon,
$$

where

$$
K_{1}=T_{g}^{\infty}-\frac{K}{M_{0}},
$$

and

$$
K_{2}=\frac{K g}{\rho A_{0}} \text {. }
$$

The linear decrease of $T_{g}$ as a function of the absorbed dose $\varepsilon$, predicted by Eq. (7), explains the trend that we observe experimentally (Fig. 5). We can further expect the transition to become more gradual with increasing $\mathrm{x}$-ray dose as the polydispersity of the polymer in the structures increases by the scissioning events that can be assumed to occur randomly along the length of the polymer backbones. Therefore, the broadening in the glass transition with increased preexposure dose observed in Fig. 4 can be interpreted as an increase in the polydispersity of the molecular weight of the PMMA. ${ }^{4}$

The theoretical formula for the linear decrease of $T_{g}$ as a function of the absorbed dose $\varepsilon$ [Eq. (7)] can be directly compared with the experimental findings by calculating the constants $K_{1}$ and $K_{2}$ using Eqs. (8) and (9) and inserting them in Eq. (7). Table I shows the properties of PMMA used in this calculation. The values for $K, g$, and $\rho$ were taken from previously reported values. ${ }^{37,39}$ The value of $118.0^{\circ} \mathrm{C}$ (with an error bar of $\pm 3.0^{\circ} \mathrm{C}$ ) for $T_{g}^{\infty}$ is the estimated value of $T_{g}$ for the sample that was not pre-exposed (see Fig. 4). The theoretically expected dependence of $T_{g}$ on the dose is thus 


$$
T_{g}\left({ }^{\circ} \mathrm{C}\right)=118.0-8.2 \times 10^{-2}-8.0 \times 10^{-3} \varepsilon\left(\mathrm{mJ} / \mathrm{mm}^{3}\right) .
$$

This dose dependence is plotted in Fig. 5. We conclude that, within the error bars, the theoretical values agree quite well with the experimental values.

In the above model we have assumed the molecular weight of the polymer forming the grating lines to be equal to that of the polymer that was spun from solution onto the $\mathrm{Si}$ wafer. The EUV-IL technique that was used to form the PMMA nanostructures, however, involved an exposure step which depends precisely on the same process, i.e., chain scission to alter the solubility of the exposed polymer in the developer solution. Therefore, the potential effect of the EUV exposure on the $T_{g}$ of the grating needs to be considered. The image in the EUV-IL exposure had a sinusoidal intensity distribution and PMMA, which is a positive resist, was removed during the development from the areas that correspond to the region near the intensity maximum. The PMMA lines that remained on the surface consequently received a minimal dose as they corresponded to the broad minima of the sinusoidal intensity distribution. The exposure dose necessary for lithographic exposure of PMMA is around $1000 \mathrm{~mJ} / \mathrm{mm}^{3},{ }^{41}$ which is of the same order as the doses that were used in the pre-exposures (Fig. 5). Therefore, we can conclude that the $T_{g}$ 's of the gratings considered in our experiments were not significantly modified by the EUV-IL fabrication process, which is also confirmed by the good agreement of the measurements with the theoretical model.

\section{CONCLUSION}

The x-ray diffraction method described here is an effective tool for measuring the glass transition temperature of nanostructured polymer films. The influence of radiationinduced damage and polymer molecular weight on the measured glass transition temperature was determined and found to be in agreement with a theoretical model. The sample that was not exposed to $\mathrm{x}$ rays prior to the diffraction measurements was found to have a $T_{g}$ equal to the one expected for the bulk polymer of the same molecular weight. While the present experiments were performed at a synchrotron radiation facility, the developed method is applicable for use with laboratory x-ray sources. The method should be especially useful in studying the effects of film thickness, nanostructure size and dimensionality (one-dimensional for lines and zerodimensional for dots), and polymer-substrate interfacial interactions on the glass transition. Nanoimprinting methods can be used to fabricate the necessary nanostructures for a large variety of polymers, such that the polymer does not have to be one that works as a photoresist like PMMA.

\section{ACKNOWLEDGMENTS}

We thank Michael Lange and Dominik Meister for help with the experimental setup at the MS beamline. Part of this work was performed at the Swiss Light Source, Paul Scherrer Institut, Villigen, Switzerland.
${ }^{1}$ J. M. G. Cowie, Polymers: Chemistry and Physics of Modern Materials, 2nd ed. (Chapman and Hall, London, 1991).

${ }^{2}$ J. L. Keddie, R. A. L. Jones, and R. A. Cory, Europhys. Lett. 27, 59 (1994).

${ }^{3}$ J. A. Forrest, K. Dalnoki-Veress, and J. R. Dutcher, Phys. Rev. E 56, 5705 (1997).

${ }^{4}$ K. Dalnoki-Veress, J. A. Forrest, C. Murray, C. Gigault, and J. R. Dutcher, Phys. Rev. E 63, 031801 (2001).

${ }^{5}$ D. S. Fryer, R. D. Peters, E. J. Kim, J. E. Tomaszewski, J. J. de Pablo, P. F. Nealey, C. C. White, and W. L. Wu, Macromolecules 34, 5627 (2001).

${ }^{6}$ R. S. Tate, D. S. Fryer, S. Pasqualini, M. Montague, J. J. de Pablo, and P. F. Nealey, J. Chem. Phys. 115, 9982 (2001).

${ }^{7}$ J. A. Forrest, K. Dalnoki-Veress, J. R. Stevens, and J. R. Dutcher, Phys. Rev. Lett. 77, 2002 (1996).

${ }^{8}$ W. E. Wallace, J. H. van Zanten, and W. L. Wu, Phys. Rev. E 52, R3329 (1995).

${ }^{9}$ D. S. Fryer, P. F. Nealey, and J. J. de Pablo, Macromolecules 33, 6439 (2000).

${ }^{10}$ C. J. Ellison and J. M. Torkelson, Nat. Mater. 2, 695 (2003).

${ }^{11}$ C. J. Ellison and J. M. Torkelson, J. Polym. Sci., Part B: Polym. Phys. 40, 2745 (2002).

${ }^{12}$ J. A. Forrest, C. Svanberg, K. Revesz, M. Rodahl, L. M. Torell, and B. Kasemo, Phys. Rev. E 58, R1226 (1998).

${ }^{13}$ J. A. Torres, P. F. Nealey, and J. J. de Pablo, Phys. Rev. Lett. 85, 3221 (2000).

${ }^{14}$ T. R. Böhme and J. J. de Pablo, J. Chem. Phys. 116, 9939 (2002).

${ }^{15}$ K. Van Workum and J. J. de Pablo, Nano Lett. 3, 1405 (2003).

${ }^{16}$ K. Yoshimoto, T. S. Jain, P. F. Nealey, and J. J. de Pablo, J. Chem. Phys. 122, 144712 (2005).

${ }^{17}$ C. M. Stafford, B. D. Vogt, C. Harrison, D. Julthongpiput, and R. Huang, Macromolecules 39, 5095 (2006).

${ }^{18}$ B. Frank, A. P. Gast, T. P. Russell, H. R. Brown, and C. J. Hawker, Macromolecules 29, 6531 (1996).

${ }^{19}$ D. B. Hall and J. M. Torkelson, Macromolecules 31, 8817 (1998).

${ }^{20}$ C. J. Ellison, S. D. Kim, D. B. Hall, and J. M. Torkelson, Eur. Phys. J. E 8, 155 (2002).

${ }^{21}$ R. D. Priestley, C. J. Ellison, L. J. Broadbelt, and J. M. Torkelson, Science 309, 456 (2005).

${ }^{22}$ S. Kawana and R. A. L. Jones, Eur. Phys. J. E 10, 223 (2003).

${ }^{23}$ International Technology Roadmap for Semiconductors (ITRS), 2005 ed. (Semiconductor Industry Association, San Jose, CA, 2005).

${ }^{24}$ M. P. Stoykovich, H. B. Cao, K. Yoshimoto, L. E. Ocola, and P. F. Nealey, Adv. Mater. 15, 1180 (2003).

${ }^{25}$ K. Yoshimoto, M. P. Stoykovich, H. B. Cao, J. J. de Pablo, P. F. Nealey, and W. J. Drugan, J. Appl. Phys. 96, 1857 (2004).

${ }^{26}$ M. K. Mundra, S. K. Donthu, V. P. Dravid, and J. M. Torkelson, Nano Lett. 7, 713 (2007).

${ }^{27}$ W. Cheng, G. Fytas, A. V. Kiyanova, M. Y. Efremov, and P. F. Nealey, Macromol. Rapid Commun. 27, 702 (2006).

${ }^{28}$ R. Hartschuh et al., J. Polym. Sci., Part B: Polym. Phys. 42, 1106 (2004).

${ }^{29}$ R. D. Hartschuh, A. Kisliuk, V. Novikov, A. P. Sokolov, P. R. Heyliger, C. M. Flannery, W. L. Johnson, C. L. Soles, and W. L. Wu, Appl. Phys. Lett. 87, 173121 (2005).

${ }^{30}$ R. L. Jones, T. Hu, C. L. Soles, E. K. Lin, R. M. Reano, S. W. Pang, and D. M. Casa, Nano Lett. 6, 1723 (2006).

${ }^{31}$ H. H. Solak, C. David, J. Gobrecht, V. Golovkina, F. Cerrina, S. O. Kim, and P. F. Nealey, Microelectron. Eng. 67, 56 (2003).

${ }^{32}$ H. H. Solak, J. Phys. D 39, R171 (2006).

${ }^{33}$ B. D. Patterson et al., Nucl. Instrum. Methods Phys. Res. A 540, 42 (2005).

${ }^{34}$ C. Broennimann et al., J. Synchrotron Radiat. 13, 120 (2006).

${ }^{35}$ H. Keymeulen, Ph.D. thesis, Swiss Federal Institute of Technology Zürich, 2006.

${ }^{36}$ Z. N. Yu, L. Chen, W. Wu, H. X. Ge, and S. Y. Chou, J. Vac. Sci. Technol. B 21, 2089 (2003).

${ }^{37}$ P. Vasudevan and M. Santappa, J. Polym. Sci., Part A-2 9, 483 (1971).

${ }^{38}$ J. S. Greeneich, J. Electrochem. Soc. 121, 1669 (1974).

${ }^{39}$ J. S. Greeneich, J. Electrochem. Soc. 122, 970 (1975).

${ }^{40}$ E. A. Dobisz, S. L. Brandow, R. Bass, and A. Mitterender, J. Vac. Sci. Technol. 18, 107 (2000).

${ }^{41}$ D. He, H. H. Solak, W. Li, and F. Cerrina, J. Vac. Sci. Technol. B 17, 3379 (1999). 